among the smallest $\mathrm{Cu}_{2-x}$ Se nanomaterials yet synthesized.

According to the researchers, cubic phase two-dimensional nanomaterials are uncommon, though a significant number occur in wet chemical syntheses. It is thought that these phases are the result of growth kinetics being controlled by ligand interactions with certain crystal planes of the materials as they grow in solution. The researchers said that the NHC products generated after selenium is removed from the precursor molecule play a significant role in forming the discshaped materials, particularly by coordi- nating copper species on one particular plane forcing continued growth to occur preferentially in the radial direction rather than equally in all directions. Changing the selenium precursor concentration relative to the copper source altered the particle shapes, which varied from a mixture of discs and tetragonal particles to irregular morphology. Using selenium powder instead of the NHC-based precursor formed irregular particles.

$\mathrm{Cu}_{2-x} \mathrm{Se}$ is a $p$-type semiconductor with a 1.0-1.4 eV indirect bandgap, a range suitable for use as an absorbing layer in photovoltaic devices. Based on UV-vis absorption, the researchers calculate the nanodisc bandgap to be $1.55 \mathrm{eV}$, an increase attributed to the quantum confinement effect. Photoconductivity measurements as a function of electrical bias on thin films of nanodiscs contacted by silver electrodes on glass substrates show an approximately sixfold increase in current under illumination compared with dark current. This is taken as evidence that the nanodiscs synthesized in this work may be incorporated into solar cells through solution-based processing.

Alia P. SCHOEN

\section{White Light Illumination Produces Tunable Color from Silicon Nanostructures}

Silicon is widely used in the microelectronics industry due to its electronic characteristics. On the other hand, the use of $\mathrm{Si}$ for optical applications requiring emission of light has been limited by the fact that silicon has an indirect bandgap, which results in poor light emission. Researchers have somewhat overcome these limitations by leveraging the impressive nanofabrication capabilities that exist for Si to produce structures capable of light emission, optical cloaking, waveguiding, and sensing.

Recently, L. Cao and colleagues from the Geballe Laboratory for Advanced Materials at Stanford University have exploited the resonant light scattering properties of Si nanostructures to produce silicon nanowires (SiNWs) and nanoparticles (SiNPs) with engineered color. In the July 14 th issue of Nano Letters (DOI: 10.1021/nl1013794; p. 2649), the team described the white light illumination (polarized and unpolarized) of SiNWs under grazing incidence using a confocal, darkfield optical microscope. The group led by M.L. Brongersma grew SiNWs using a gold colloid-catalyzed chemical vapor deposition process which allowed for accurate diameter control and precise elucidation of the relation-

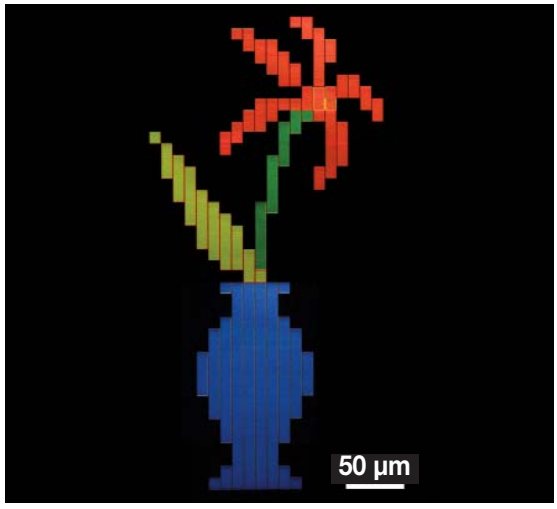

Figure 1. Darkfield optical image of a large-scale patterned Si nanowire (SiNW) array. The array was fabricated using standard polycrystalline silicon thin-film deposition, lithographic patterning, and dry etching. The pattern consists of four types of arrays with distinct NW widths: $170 \mathrm{~nm}$ (blue), $200 \mathrm{~nm}$ (green), $240 \mathrm{~nm}$ (yellow), and $300 \mathrm{~nm}$ (red). The NW lengths $(10 \mu \mathrm{m})$ were chosen to be much longer than the width. Their height was set by the deposited film thickness of $180 \mathrm{~nm}$. The interspacing between NWs was $130 \mathrm{~nm}$. Credit Pengyu Fan

ship between NW size and resonant light scattering. The researchers observed a continuous change in SiNW color across the entire visible spectrum under ran- domly polarized white light illumination, with color shifting linearly from blue to red with increasing wire diameter. Similarly, they observed size-dependent color in large-scale, sub-wavelength SiNW and SiNP arrays (Figure 1) which were generated with carefully selected physical dimensions using standard polycrystalline Si thin-film deposition, lithographic patterning, and dry etching.

The Stanford team noted that color also varied with the polarization state of the illumination source and related this polarization dependence, as well as the size dependence, to the well-known Lorentz-Mie theory of light scattering. They also described the sensitivity of SiNW light scattering to their surrounding dielectric environment, which is related to the "leaky" nature of NW modes whose fields extend outside of the NWs. The ability to engineer color in Si nanostructures by strategic selection of size, dielectric environment, and illumination conditions greatly increases its potential application for information, display, camouflage, ornamental, and biotechnologies.

"Si is expected to further solidify its dominance in an increasing number of high-volume technologies," said Cao, when considering these new findings in conjunction with Si's excellent electrical, mechanical, and biochemical properties. "This puts this material in a new light."

SAMESHA R. BARNES

\section{Stagnation Mechanism Revealed in Simulations of Grain-Boundary Roughening in Nickel at High Temperatures}

Grain growth in polycrystalline materials almost always stops well before the equilibrium state of single crystal is reached. Conventional mechanisms for grain-growth stagnation include solute segregation to grain boundaries and pinning of grain boundaries by second phase particles. However, grain-growth stagnation is also observed in materials where such mechanisms are not applicable, as in high purity materials, and materials with high solute diffusivity. In order to expand understanding of the mechanisms hindering grain growth, E.A. Holm and S.M. Foiles from
Sandia National Laboratories applied computational models to simulate grain growth in pure nickel. Their results suggested an alternative mechanism, which they described in the May 28th issue of Science (DOI: 10.1126/science.1187833; p. 1138).

The researchers used a synthetic-driving force molecular dynamics method (MD) to calculate grain boundary mobility for a 
catalog of 388 individual grain boundaries in pure nickel. At a given temperature they found that each grain boundary was either a smooth atomic-structured boundary with very low mobility, or rough atomic-structured boundaries with high mobility. After reaching a transition temperature, which differs from boundary to boundary, the grain boundary structure changes from smooth to rough and the mobility decreases. Holm and Foiles applied these data to a mesoscale simulation for a polycrystalline microstructure using the Monte Carlo Potts model for grain growth. When smooth/slow bound- aries are present, the researchers found that grain growth plateaus at a finite grain size value which decreased as the fraction of smooth/slow boundaries increases. In addition, they found that stagnant grain size increases with temperature. These results suggested that the boundary roughening transition could influence grain growth stagnation.

Holm and Foiles performed another MD simulation, a grain growth in a realistic three-dimensional nanocrystalline nickle grain structure. At the temperatures analyzed, simulation results showed that after a short initial transient state, normal grain growth occurs. However, after some time, grain growth slows and grain size appears to stagnate. Moreover, the stagnant grain sizes observed were consistent with results from mesoscale simulations. Simulation results from different methods demonstrated that the presence of atomically smooth grain boundaries can stop in the absence of solute or pinning particles grain growth. Holm and Foiles said that atomically smooth grain boundary structures provide an alternative mechanism for grain-growth stagnation at high temperatures for pure materials.

SIARI S. SOSA

\section{All-Quantum-Dot Multilayer LEDs Prepared Using Layer-by-Layer Solution Processing Show High Brightness}

The quest for cheap and energy-efficient lighting and light-emitting displays has resulted in robust efforts to develop technologies for light-emitting diodes (LEDs). The use of quantum dots (QDs) in LEDs offers the advantages of high color purity and easy tunability of emission. High brightness has been achieved with very thin QD layers (one-to-two monolayers), but challenges in research on QD LEDs remain. Increasing the devices' lifetime, brightness, and homogeneous deposition over large areas, as well as avoiding the use of toxic materials, would make them serious alternatives to organic devices. W.K. Bae, K.H. Char, C.H. Lee, S.H. Lee, and co-workers from the Seoul National University have recently demonstrated the large-area deposition of multicolored allQD (QDs with core and chemical composition gradient shell structure-mostly CdSe/ZnS) LEDs prepared by the spinassisted layer-by-layer assembly method.

As reported in the July 14th issue of Nano Letters (DOI: 10.1021/nl100168s; p. 2368 ), the research team fabricated allquantum-dot LEDs with well-controlled vertical and horizontal structures. Quantum dots were treated with cysteamine (CAm) or mercaptopropionic acid (MPA) and dispersed in water to obtain positive and negative charges on the surface of the nanocrystals. Alternate spincoating depositions of positively and negatively charged nanocrystals allowed the build-up of multilayered thin films con-
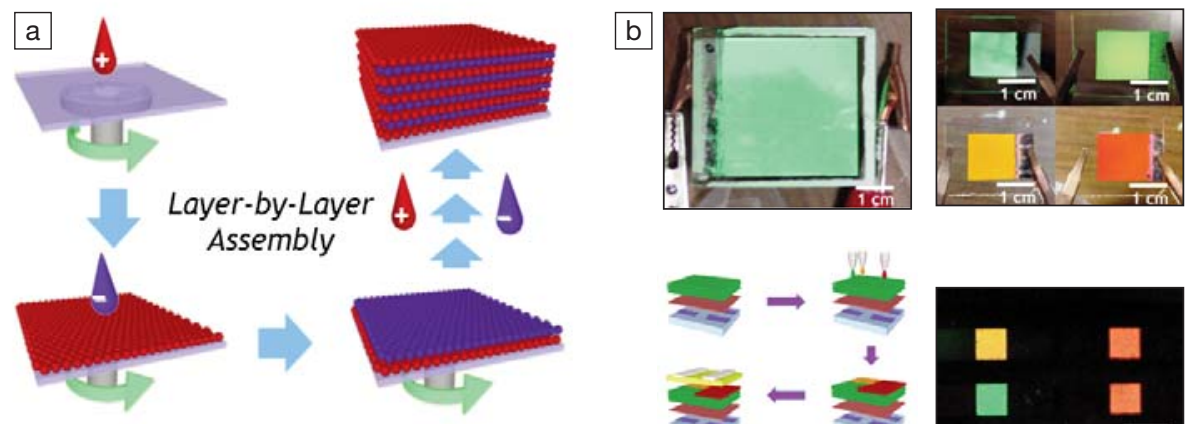

All-QD Multilayer Film
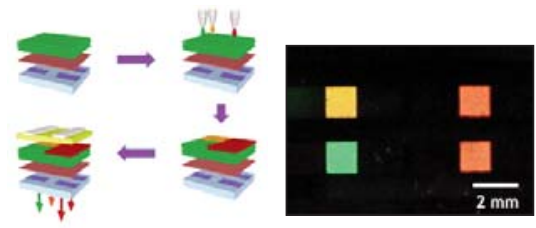

Figure 1. Preparation of all-quantum-dot (QD) multilayer films by (a) spin-assisted layer-bylayer deposition of oppositely charged monolayers; and (b) multicolored-QD light-emitting diodes in large area arrays with schematics of deposition procedure obtained by variation of the nature of the nanocrystals in the exciton recombination zone.

sisting of submonolayers of nanocrystals (i.e., QD-CAm/QD-MPA $n$ ). Such deposition techniques permit the facile scale-up to larger device area of homogeneous and uniform films.

The researchers could, with this processing method, control the vertical multilayered morphology of their films. In order to figure out where an exciton recombination occurred, they integrated a single or double layer of green-emitting nanocrystals located at different vertical positions in thin films comprised primarily of layers of red-emitting nanocrystals. They studied the electroluminescence of these various multilayered thin films and found that it mostly arises from the top layer, adjacent to the electron transporting layer-made of $40 \mathrm{~nm}$ of $2,2^{\prime}, 2^{\prime \prime}-(1,3,5-$
benzinetryil)tris(1-phenyl-1-H-benzimidazole) that is, TBPi. The vacancies in the first submonolayer induced a small electroluminescent contribution $(\sim 10 \%)$ from the second-to-the-top layer. This is consistent with the previous optimized architecture consisting of one or two monolayers of quantum dots.

The researchers coupled the spin-assisted layer-by-layer assembly to patterning techniques compatible with solution processing, thus also controlling the horizontal morphology of devices. By using nanocrystals of different sizes, they realized three-colored LEDs fabricated from allquantum dot multilayers with the potential for full-color, larger area deposition and device characteristics improvements.

ELSA COUDERC 